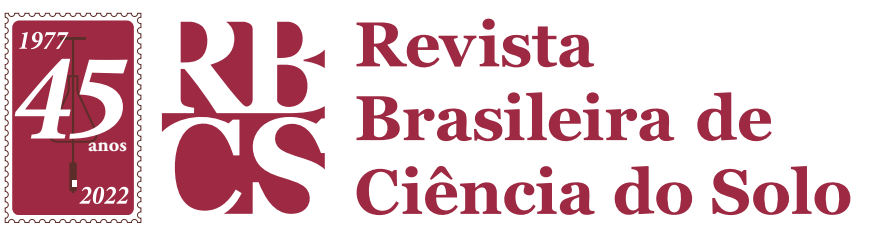

Division - Soil Processes and Properties | Commission - Soil Biology

\title{
Effects of Meloidogyne incognita on the fungal community in tobacco rhizosphere
}

\author{
Chen Xiaolong $^{(1)}\left(\mathbb{D}\right.$, Gao Lingling ${ }^{(2)}\left(\mathbb{D}\right.$, Deng Xiaopeng ${ }^{(3)}(\mathbb{D})$ Yang Yongfeng $^{(1)}(\mathbb{D}$, \\ Wang Jianwei $^{(4)}\left(\mathbb{D}\right.$, Zhang Zhan ${ }^{(1)}$ (D) Cai Yongzhan ${ }^{(5)}\left(\mathbb{D}\right.$, Huang Feiyan ${ }^{(6)}(\mathbb{D}$, Yang \\ Min $^{(6)}$ (iD) Tong Wenjie ${ }^{(3)^{*}}$ (iD) and Yu Lei ${ }^{(6)}$ (iD) \\ (1) Tobacco Leaf Purchase Center, China Tobacco Henan Industrial Co., Ltd., Henan, China. \\ (2) Center of Agricultural Products Processing, Henan Academy of Agricultural Sciences, Henan, China. \\ (3) Yunnan Academy of Tobacco Science, Yunnan, China. \\ ${ }^{(4)}$ Zhengzhou Tobacco Research Institute of China National Tobacco Corporation, Henan, China. \\ (5) Yunnan Provincial Tobacco Company Qujing Branch, Yunnan, China. \\ ${ }^{(6)}$ Kunming University, Yunnan Urban Agricultural Engineering \& Technological Research Center, Yunnan, \\ China.
}

* Corresponding author:

E-mail: 376914788@qq.com

Received: September 17, 2021

Approved: November 16, 2021

How to cite: Xiaolong $C$,

Lingling $\mathrm{G}$, Xiaopeng $\mathrm{D}$, Yongfeng $Y$, Jianwei W, Zhan Z, Yongzhan

C, Feiyan $\mathrm{H}$, Min Y, Wenjie T, Lei $Y$ Effects of Meloidogyne incognita on the fungal community in tobacco rhizosphere. Rev Bras Cienc Solo. 2022;46:e0210127.

https://doi.org/10.36783/18069657rbcs20210127

Editors: José Miguel Reichert (ID and Juvenil Enrique Cares (D).

Copyright: This is an open-access article distributed under the terms of the Creative Commons Attribution License, which permits unrestricted use, distribution, and reproduction in any medium, provided that the original author and source are credited.
ABSTRACT: The often widespread and serious Root-Knot Nematode (RKN) disease is an important soil-borne disease affecting tobacco production. This study aimed to understand micro-ecological changes caused by RKN disease and interactions between disease and rhizosphere soil fungal communities. The 18S rRNA gene sequencing was used to study changes in rhizosphere fungal community of tobacco plants having RKN disease. In June 2018, a paired comparison was performed between rhizosphere fungal community structures of healthy tobacco plants and those with RKN disease in Yuxi and Jiuxiang, Yunnan Province, China. Compared with uninfested soil, the OTU abundance, Shannon, ACE and Chaol indexes of infested soil in the two tobacco areas showed a decreasing trend. Principal Coordinate Analysis showed fungal communities of infested soil and uninfested soil in the two tobacco areas were clustered in different areas, and the community composition was significantly different. Moreover, the dominant fungi community and relative abundance are significantly different at phylum, genus and species levels. More beneficial fungi, such as Penicillium and Aspergillus, were found in soil samples of healthy plants, whereas more pathogenic fungi, such as Phoma and Alternaria, were found in soil samples of diseased plants. In conclusion, changes in fungal community structure and decreases in species diversity and richness were important characteristics of rhizosphere soils from diseased tobacco plants. Disequilibrium in the tobacco rhizosphere micro-ecosystem may allow the development of RKN disease and other more complex diseases.

Keywords: root-knot nematode disease, soil-borne disease, soil microorganism, diversity. 


\section{INTRODUCTION}

Meloidogyne spp. is an important group of plant parasitic nematodes, having many species and a wide host range (Back et al., 2002; Jeger et al., 2018). Members including Meloidogyne incognita, Meloidogyne hapla, Meloidogyne javanica and Meloidogyne arenaria are causal agents of tobacco RKN disease, an important soil-borne disease that negatively impacts tobacco production. This desease occurs in 13 major tobacco producing areas in Yunnan, Henan and Shandong provinces of China. For example, in Yunnan, more than 26,000 hectare is affected by the disease, resulting in yield losses of 30 to $50 \%$ (Chen et al., 2015). Furthermore, the Meloidogyne spp. often infect roots and cause mechanical damage, and the tiny wounds greatly assist in infection by other pathogenic microorganisms, such as Ralstonia solanacearum and Phytophthora (Cui et al., 2021). Multiple infections form complex infectious diseases that seriously affect the yield and quality of tobacco leaves. Flue-cured tobacco is an important economic crop in Yunnan, which contains one-third of the total planting area, and produces a corresponding yield in China. Thus, this region plays an important role in China's tobacco industry (Tong et al., 2016). The RKN disease has become an important factor restricting the development of the Yunnan tobacco industry.

Occurrences of soil borne diseases are closely related to imbalances in soil micro-ecosystems (Liu et al., 2016; Kim and Anderson, 2018). Plant rhizosphere microorganisms and soil-borne pathogens grow in the same environment; consequently, their competition for limited resources, such as nutrients, and niches directly or indirectly affects the occurrence and development of plant diseases (Li and Wang, 1989). Some parasitic, symbiotic or antagonistic relationship exists between Meloidogyne spp. and soil microorganisms, and some microbial communities have inhibitory effects on Meloidogyne spp. (Ruby et al., 2008; Haegeman et al., 2009). At present, Meloidogyne parasitoids and antagonistic bacteria have been identified in Bacillus, Pseudomonas, Streptomyces and Pasteuria (Siddiqui et al., 2002; Tian et al., 2015). For example, Pasteuria penetrans has a highly specific parasitic capability and is an effective biocontrol agent against $M$. incognita and M. javanica (Stirling et al., 2011; Ciancio et al., 2016). Pseudomonas can inhibit Meloidogyne spp. by destroying their eggs, reducing their hatching levels and specifically activating peroxidase and phenylalanine ammonia lyase (Norabadi et al., 2014; Cao et al., 2015).

Furhtermore, some fungi can also inhibit Meloidogyne spp. through predation or parasitism. More than 30 parasitic fungi of root-knot nematode have been reported (Yang et al., 2004); for example, the hyphae of Paecilomyces lilacinus and Pochonia chlamydosporia can enter Meloidogyne eggs through parasitism and digest and absorb the substances in the eggs, which leads to abnormal egg development (Liu, 2011). Other species such as Coprinus comatus, Gliocladium roseum, Trichoderma harzianum, Trichoderma viride can also effectively inhibit Meloidogyne spp. (Yang et al., 2004; Cui et al., 2021). Although these previous studies precisely and comprehensively illustrated the involvement of soil microbes in antagonistic interaction with plant-parasitic nematodes, studies identifying the specific group of soil fungus associated with the occurrence of nematodes in the field are still limited (Huang et al., 2020).

Life cycle and development of most plant-parasitic nematodes occur in the rhizosphere of host plants. Rhizosphere is not only the window of efficient utilization of crop nutrients, but also the vector of successful infection of pathogens. It is the key micro domain of plant-soil-microorganism interaction (Yang et al., 2019). In the same plot, with similar natural conditions and soil properties, the existence of both Meloidogyne incognita -infected and uninfected plants indicates that there may be specific interactions between plants and rhizosphere soil micro ecological environment, which directly or indirectly affect the occurrence of diseases (Ruby et al., 2008). 
We hypothesize that there may be some specific microorganisms regulating Meloidogyne spp. activities in the soil. Therefore, Illumina MiSeq sequencing was used to analyze the differences in the fungal community in rhizosphere soil between healthy tobacco plants and those with RKN disease. This study aimed to explore the relationship between the structure and diversity of the fungal community in tobacco rhizosphere soil and RKN disease and provide a scientific basis for the effective prevention and control of RKN disease in tobacco.

\section{MATERIALS AND METHODS}

\section{Research area}

The experiment was conducted on Gaocang Street, Hongta District, Yuxi City, Yunnan $\left(24^{\circ} 30^{\prime} \mathrm{N}, 103^{\circ} 32^{\prime} \mathrm{E}\right)$ and Jiuxiang Township, Yiliang County, Kunming City, Yunnan $\left(25^{\circ} 1^{\prime} 43^{\prime \prime} \mathrm{N}, 103^{\circ} 20^{\prime} 31^{\prime \prime} \mathrm{E}\right)$ in June 2018. Average annual rainfall in the Hongta District of Yuxi City is $779.5-989.7 \mathrm{~mm}$. The average annual temperature is $15.6-23.8^{\circ} \mathrm{C}$; the annual frost-free period is $244-365$ days; the average annual sunshine is $2,115-2,285 \mathrm{~h}$. The altitude of the site is $1,760 \mathrm{~m}$, the soil type is red loam soil, and the irrigation method is hole irrigation. The previously planted crop was wheat, the land is relatively flat, and the drainage is good.

The average annual rainfall in Jiuxiang Township Yiliang County of Kunming City is 526.9-898.9 $\mathrm{mm}$, the annual average temperature is $16.3-21.7^{\circ} \mathrm{C}$, the annual frost-free period is about $260 \mathrm{~d}$, and the annual average sunshine is approximately $2,032 \mathrm{~h}$. The altitude of the site is $1,560 \mathrm{~m}$, the soil type is red loam soil, the irrigation method is hole irrigation, and the previous crop was corn. Table 1 shows the basic soil properties of the 0.00-0.20 $\mathrm{m}$ soil layer in the two test plots before plowing.

After being suspended with water (soil:water ratio $=1: 2.5$ ), the soil $\mathrm{pH}$ was measured using a pH meter; organic matter with the acidified potassium dichromate $\left(\mathrm{K}_{2} \mathrm{Cr}_{2} \mathrm{O}_{7}-\mathrm{H}_{2} \mathrm{SO}_{4}\right)$ heating method; total nitrogen was measured by Dumas Nitrogen Analyzer, available phosphorus was determined with $\mathrm{NaHCO}_{3} 0.5 \mathrm{~mol} \mathrm{~L}^{-1}(\mathrm{pH} 8.5)$; available potassium was determined by $\mathrm{CH}_{3} \mathrm{COONH}_{4}$ extraction method.

\section{Materials and soil sampling}

Tobacco fields with typical and serious RKN disease (Meloidogyne incognita) levels were selected in the test areas. In the same plot, with similar conditions and soil properties, plants with typical symptoms of RKN disease ( $M$. incognita) and healthy plants were sampled, with three plants per group. The topsoil and whole plants were removed. The loose soil was shaken off, and the soil attached to the root at 0-4 mm was collected as rhizosphere soil. The samples were named as jiuxiang infested soil (JX_P), Jiuxiang uninfested soil (JX_N), Yuxi infested soil (YX_P) and Yuxi uninfested soil (YX_N). After removing the impurities and residual fine roots, the soil samples were put separately into sterile self-sealed bags, immediately placed in liquid nitrogen, transported back to the laboratory, and stored at $-80^{\circ} \mathrm{C}$.

Table 1. Basic soil properties of the $0.00-0.20 \mathrm{~m}$ soil layer before plowing

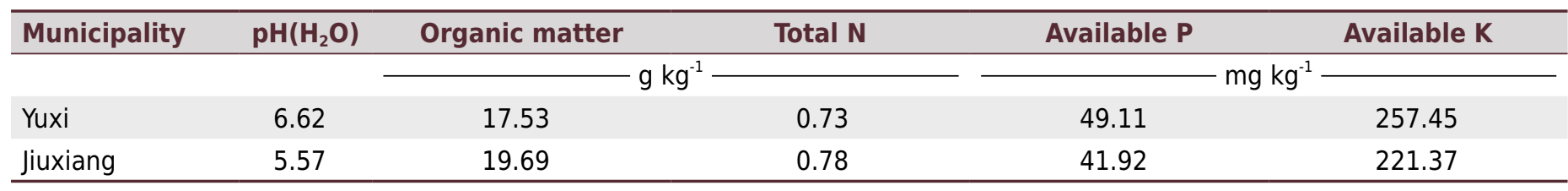




\section{DNA extraction and PCR amplification of fungi}

Total DNA in the infested and uninfested soil of the two test sites (JX_P, JX_N, YX_P, YX_N) was extracted following the instructions of a FastDNA ${ }^{\circledR}$ SPIN Kit for Soil (USA). The DNA concentration and purity were detected using a NanoDrop2000, and the DNA quality was determined using $1 \%$ agarose gel electrophoresis.

Primers ITS1 F (5'-ACTTGGTCATTTAGGAAGTAA-3') and ITS2 R (5'-BGCTGCGTTCTTCATCGATGC-3') were used to amplify the ITS- 1 variable region of the $18 \mathrm{~S}$ rRNA. The amplification procedure was as follows: pre-denaturation at $95^{\circ} \mathrm{C}$ for 3 min, 36 cycles of denaturation at $95^{\circ} \mathrm{C}$ for $30 \mathrm{~s}$, annealing at $55^{\circ} \mathrm{C}$ for $30 \mathrm{~s}$ and extension at $72{ }^{\circ} \mathrm{C}$ for $45 \mathrm{~s}$, followed by a final extension at $72{ }^{\circ} \mathrm{C}$ for $10 \mathrm{~min}$. The amplification system was as follows: $4 \mu \mathrm{L} 5 \times$ FastPfu buffer, $2 \mu \mathrm{L} 2.5 \mathrm{mmol} \mathrm{L}^{-1} \mathrm{dNTPs}, 0.8 \mu \mathrm{L}$ forward primer $\left(5 \mu \mathrm{mol} \mathrm{L}^{-1}\right), 0.8 \mu \mathrm{L}$ reverse primer $\left(5 \mu \mathrm{mol} \mathrm{L}^{-1}\right), 0.4 \mu \mathrm{L}$ FastPfu polymerase, $0.2 \mu \mathrm{L} \mathrm{BSA}, 10 \mathrm{ng}$ DNA template and water was added until achieve a final volume of $20-\mu \mathrm{L}$.

The PCR products were recovered by $2 \%$ agarose gel electrophoresis and further purified using an AxyPrep DNA Gel Extraction Kit (Axygen, USA). Then, they were sequenced by Shanghai Meiji Biological Medicine Technology Co., Ltd using MiSeq.

\section{Sequence quality control and analysis}

The original sequence was spliced using FLASH (Caporaso et al., 2011) software. They were then filtered using Usearch (Edgar, 2013), and the chimeric sequences were removed to obtain the effective sequences. The operational taxonomic units (OTUs) were clustered using UPARSE at a $97 \%$ sequence similarity level. The sequences were annotated by RDP classifier (Wang et al., 2007) and the Silva database (Altschul et al., 1990), and the dilution curve was made using Mothur. The library coverage, Shannon, Simpson, ACE and Chaol indexes were calculated, and the species diversity and richness indexes were evaluated. A principal coordinate analysis was performed with a Bray-Curtis dissimilarity matrix using Qiime. The rarefaction curves, Veen and community composition histogram and heatmap were generated from the R software.

\section{Data analyses}

Differences in the microbial diversity index were compared using Analysis of Variance (ANOVA, IBM SPSS 22.0). Significance was determined at the $p<0.05$ level and at the $95 \%$ confidence level by Duncan's test.

\section{RESULTS}

\section{OTU abundance and the fungal alpha-diversity level}

The OTU abundance and alpha-diversity of fungal communities in different samples are shown in table 2 . Shannon index dilution curve (Figure 1) showed that the slope of the curve gradually decreased and tended to flatten as the sequencing quantity increased, indicating that the sequencing quantity was sufficient. In this experiment, the coverage rates of the four samples were greater than $99.0 \%$ (Table 2 ), indicating that the sequencing lengths were sufficient.

Variation trends in the OTU abundances from tobacco rhizosphere soil at different sampling sites were the same, having the following order: $M$. incognita infested soil $<$ uninfested soil (Table 2). Among them, the fungal OTU abundance of JX_P was $6.63 \%$ lower than that of JX_N, and the OTU abundance of YX_P was $17.60 \%$ lower than that of YX_N. The Venn diagram of OTU abundance levels of fungi is shown in figure 2 . The number of OTUs shared by JX_N and JX_P was only 587, accounting for $17.96 \%$ of the total OTUs $(3,268)$. The unique number of OTUs in JX_N was 1.43 times that of JX_P. The common 


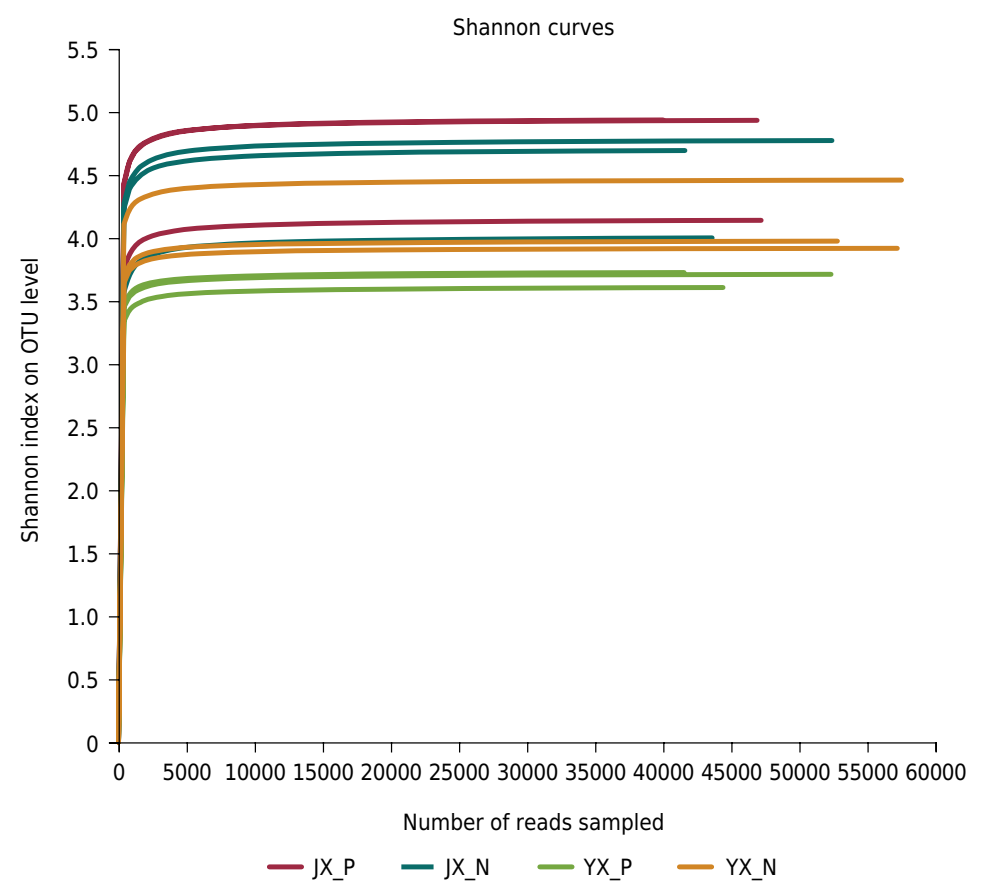

Figure 1. Shannon index-based rarefaction curves on fungus in rhizosphere soils at different samples.

Table 2. Fungus OTU abundance and alpha diversity index in rhizosphere soils at different samples

\begin{tabular}{|c|c|c|c|c|c|c|c|c|}
\hline \multirow{2}{*}{ Samples } & \multirow{2}{*}{ Raw number } & \multirow{2}{*}{ Effective number } & \multirow{2}{*}{ OTU abundance } & \multicolumn{4}{|c|}{ Alpha diversity } & \multirow{2}{*}{ Coverage } \\
\hline & & & & Shannon & Simpson & ACE & Chao1 & \\
\hline & & & & & & & & $\%$ \\
\hline JX_N & 48510 & 45741 & 1690 & $4.80 \mathrm{a}$ & $0.0218 b$ & $1307.30 \mathrm{a}$ & $1281.67 \mathrm{a}$ & 99.39 \\
\hline JX_P & 47215 & 44583 & 1578 & $4.36 \mathrm{ab}$ & $0.0563 a b$ & $1205.42 \mathrm{a}$ & $1190.45 \mathrm{a}$ & 99.46 \\
\hline YX_N & 58228 & 55764 & 1193 & $4.12 b c$ & $0.0379 a b$ & $1023.62 \mathrm{~b}$ & $984.02 \mathrm{~b}$ & 99.58 \\
\hline$Y X \_P$ & 47849 & 45875 & 983 & $3.68 \mathrm{c}$ & $0.0669 \mathrm{a}$ & $958.86 \mathrm{~b}$ & $953.19 \mathrm{~b}$ & 99.51 \\
\hline
\end{tabular}

Different lowercase letters indicate a significant difference between treatments $(p<0.05)$.

OTUs (223) in YX_N and YX_P accounted for $10.25 \%$ of the total OTUs $(2,176)$. The unique number of OTUs in YX_N was 2.11 times that of $Y X \_P$, indicating that the occurrence of RKN disease significantly reduced the OTU abundance of the fungi.

Diversity and richness of fungal communities in tobacco rhizosphere soils from the sample sites also showed the same changing trends, which were in the following order: infested soil < uninfested soil. The Shannon index of JX_P was $9.23 \%$ lower than that of JX_N, whereas that of YX_P was $10.59 \%$ lower than that of YX_N. Compared with JX_N and YX_N, the ACE indexes of JX_P and YX_P decreased by 7.79 and $6.33 \%$, respectively, and the Chaol indexes decreased by 7.12 and $3.13 \%$, respectively. In general, RKN disease has a certain impact on the OTU abundance, as well as the diversity and richness, of the fungal community in the rhizosphere soil of flue-cured tobacco, and the same change patterns occurred among the different regions.

\section{Community species and relative abundances of fungi}

Fungi relative abundance at the phylum level is shown in figure 3. Fungi in different rhizosphere soils were mainly composed of Ascomycota, Mortierellomycota and Basidiomycota at the phylum level. The relative abundances of JX_P and JX N in Ascomycota and Mortierellomycota were not significantly different. However, the relative abundance of Ascomycota in YX_P was $12.64 \%$ lower than in YX_N, whereas the relative abundance 
of Mortierellomycota in YX_P was $50.03 \%$ higher than in YX_N. The relative abundances of Basidiomycota in all different test sites followed the trend infested soil $>$ uninfested soil. JX_P had $25.39 \%$ more Basidiomycota than JX_N, and YX_P had $49.73 \%$ more Basidiomycota than YX_N.

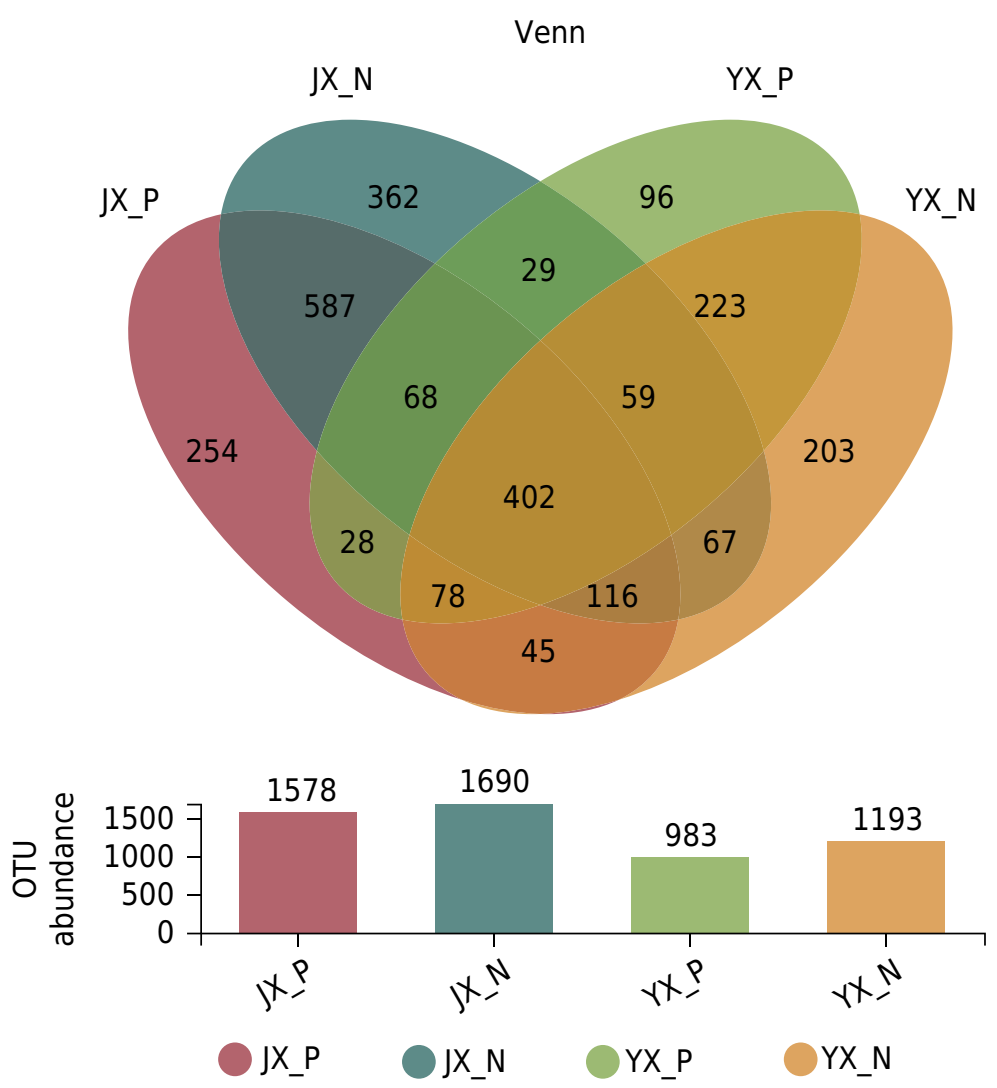

Figure 2. Venn graph of fungus OTUs distribution in rhizosphere soils at different samples.

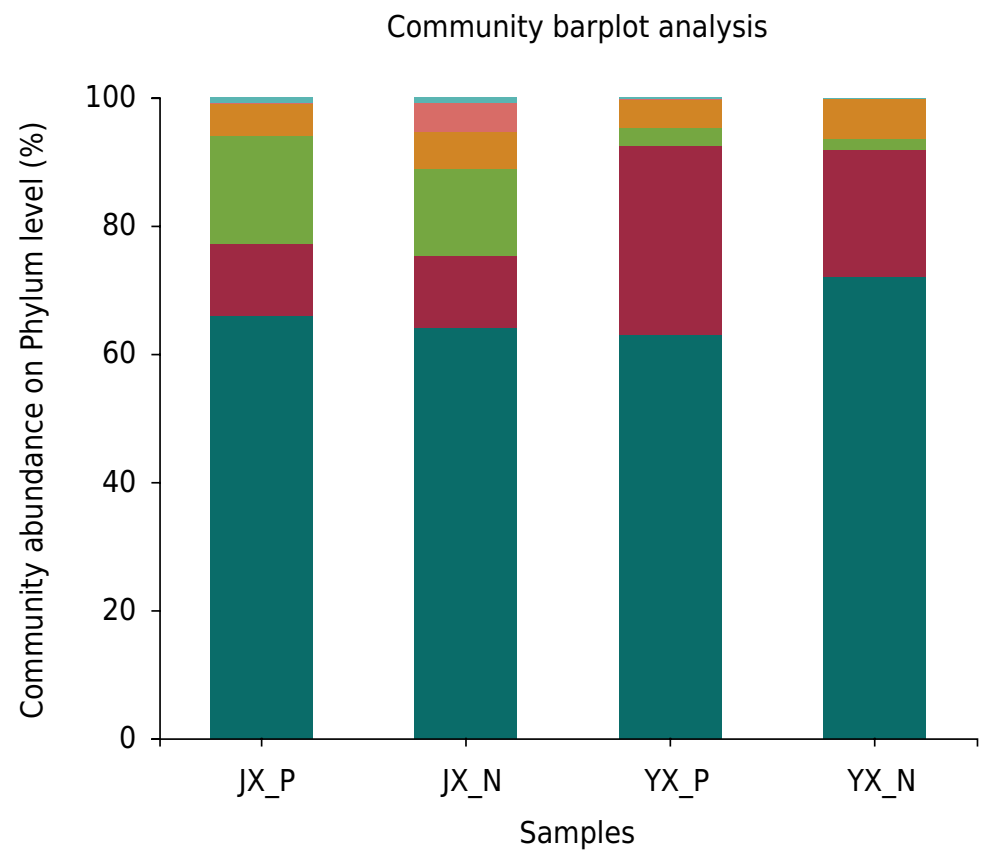

ascomycota $\square$ Mortierellomycota $\square$ Basidiomycota

nunclassified_k_Fungi Olpidiomycota nothers

Figure 3. The relative abundance of fungus on Phylum level in rhizosphere soils at different samples. 
Fungi in different samples also showed significant differences at the genus level (Table 3). Mortierella and Fusarium accounted for the largest proportions in all the samples, and their relative abundances in JX_P decreased by 1.19 and $17.42 \%$, respectively, compared within JX_N. Relative abundance of Fusarium in YX_P was $11.44 \%$ lower than in YX_N, but that of Mortierella increased by $54.35 \%$ in YX_P compared with YX_N. In addition, the relative abundances of Penicillium and Aspergillus have followed the order of uninfested soil $>$ infested soil. In JX_P, their relative abundances were 29.11 and $4.21 \%$ lower, respectively, than in JX_N; whereas in YX_P, they were 36.48 and $56.98 \%$ lower, respectively, than in YX_N. On the contrary, the relative abundances of Phoma and Alternaria in uninfested soil were lower than in infested soil. The relative abundances in JX_P were 53.57 and $1156.52 \%$ higher, respectively, than in JX_N, whereas in YX_P, they were 145.12 and $323.08 \%$ higher, respectively, than in YX_N.

Relative abundances of the top 50 species, according to the annotation and relative abundance of each sample, were selected to generate a heatmap (Figure 4). Among them, the fungi in Phoma were mainly Phoma omnivirens. Its relative abundance in different samples was as follows: JX_P $>$ JX_N $>Y_{-} X_{-} P_{-}>Y_{-} X_{-}$. Purpureocillium lavendulum relative abundance in different samples was as follows: $Y X_{-} P>Y X_{-} N>J X_{-} P>J X_{-} N$. Our results showed that the occurrence of RKN disease had a great influence on the fungal distribution and community composition in tobacco rhizosphere soil.

\section{Principal coordinate analysis of the fungal communities}

Principal component 1 (PC1) and principal component 2 (PC2) accounted for 50.53 and $11.23 \%$ of the sample differences, respectively, from $61.76 \%$ of the soil samples (Figure 5). The fungal communities of the four samples were separately distributed in the four quadrants, which revealed that the fungal communities in the different samples were quite varied. First, JX_P and JX_N were mainly distributed on the left side of the PC2 axis, whereas $Y X_{-} P$ and $Y X_{-} N$ were mainly distributed on the right side of the $P C 2$ axis. Additionally, the distance between the clusters was great, indicating that each fungal community's composition was significantly different in the experimental areas.

However, the fungal compositions in RKN infested and uninfested soil showed the same change trends in different experimental areas. Both JX_P and YX_P were concentrated on the upper side of the PC1 axis, whereas JX_N and YX_N were clustered in the lower side of the $\mathrm{PCl}$ axis, indicating that the fungal community compositions in RKN diseased and healthy soils were significantly different, but different regions had the same change trends.

\section{DISCUSSION}

Soil microbial communities are influenced by multiple factors such as plant type, climate, soil properties and agricultural practice (Bowen et al., 2017). The results of this study also confirm this point, the fungal communities in the rhizosphere soils of the jiuxiang and Yuxi experimental sites were distributed on both sides of the PC2 axis, and the distance between them was relatively great, indicating that there was a large difference in the fungal communities at the two experimental sites due to the differences in physical and chemical soil properties, climatic and cultivation management measures (Zhang et al., 2016; Tong et al., 2021). But it is worth noting that the fungal community compositions in rhizosphere soils from diseased and healthy plants in the two experimental plots showed the same trends. The fungal communities in the rhizosphere soils of diseased plants from the two experimental plots clustered on the upper side of the PC1 axis, whereas those of healthy plants clustered on the lower side of the PC1 axis, which supported that RKN disease probably led to the changes in soil microbial community. However, rhizosphere microbial community structure variations may also make the root more susceptible to pathogen infection and RKN disease. 
Table 3. The relative abundances of fungus on genus level in rhizosphere soils at different samples

\begin{tabular}{|c|c|c|c|c|}
\hline \multirow{2}{*}{ Taxonomic category (genus) } & \multicolumn{4}{|c|}{ Fungi abundance } \\
\hline & JX_P & JX_N & YX_P & YX_N \\
\hline & \multicolumn{4}{|c|}{ $\%$} \\
\hline Mortierella & 10.76 & 10.89 & 29.25 & 18.95 \\
\hline Fusarium & 4.22 & 5.11 & 11.15 & 12.59 \\
\hline unclassified_f_Chaetomiaceae & 2.86 & 3.53 & 10.72 & 9.85 \\
\hline unclassified_k_Fungi & 5.03 & 5.81 & 4.46 & 6.24 \\
\hline unclassified_c_Sordariomycetes & 2.00 & 2.47 & 5.77 & 3.74 \\
\hline Penicillium & 2.46 & 3.47 & 2.42 & 3.81 \\
\hline unclassified_p_Ascomycota & 1.82 & 4.95 & 1.94 & 2.78 \\
\hline Phoma & 3.87 & 2.52 & 2.01 & 0.82 \\
\hline Talaromyces & 2.78 & 6.22 & 0.02 & 0.06 \\
\hline unclassified_f_Stephanosporaceae & 0.09 & 8.95 & 0.03 & 0.05 \\
\hline unclassified_f_Nectriaceae & 1.19 & 2.96 & 1.46 & 2.51 \\
\hline unclassified_f_Microascaceae & 0.79 & 1.30 & 3.32 & 2.09 \\
\hline Rhodotorula & 7.51 & 0.07 & 0.03 & 0.05 \\
\hline Zopfiella & 3.13 & 3.63 & 0.35 & 0.14 \\
\hline Cercophora & 0.04 & 0.15 & 1.81 & 4.01 \\
\hline Aspergillus & 1.82 & 1.9 & 0.37 & 0.86 \\
\hline unclassified_f_Didymellaceae & 4.36 & 0.23 & 0.22 & 0.13 \\
\hline Plectosphaerella & 0.44 & 1.08 & 1.04 & 1.79 \\
\hline Trichoderma & 2.24 & 1.92 & 0.23 & 0.27 \\
\hline Olpidium & 0.05 & 4.4 & 0.02 & 0.03 \\
\hline Chaetomium & 0.82 & 0.22 & 1.06 & 1.59 \\
\hline unclassified_o_Pleosporales & 1.68 & 0.91 & 0.44 & 0.73 \\
\hline Thielaviopsis & 0.03 & 0.02 & 0.02 & 3.13 \\
\hline Cladosporium & 2.92 & 0.37 & 0.38 & 0.19 \\
\hline Cladorrhinum & 2.37 & 1.52 & 0.05 & 0.12 \\
\hline Alternaria & 2.89 & 0.23 & 0.55 & 0.13 \\
\hline Geminibasidium & 2.50 & 1.24 & 0.02 & 0.11 \\
\hline unclassified_o_Microascales & 0.65 & 0.46 & 1.58 & 0.83 \\
\hline Purpureocillium & 0.24 & 0.03 & 2.28 & 0.81 \\
\hline Metarhizium & 0.69 & 0.54 & 0.59 & 1.29 \\
\hline unclassified_o_Sordariales & 0.58 & 0.40 & 1.77 & 0.38 \\
\hline Knufia & 0.19 & 0.03 & 1.20 & 1.41 \\
\hline Guehomyces & 2.47 & 0.54 & 0.03 & 0.13 \\
\hline Arthrographis & 0.54 & 1.29 & 0.05 & 0.77 \\
\hline Ochrocladosporium & 0.04 & 0.02 & 0.02 & 0.12 \\
\hline Thielavia & 0.03 & 0.02 & 1.08 & 0.84 \\
\hline Phialemoniopsis & 0.03 & 2.12 & 0.01 & 0.16 \\
\hline Lophiostoma & 0.02 & 1.55 & 0.01 & 0.29 \\
\hline Epicoccum & 1.52 & 0.23 & 0.02 & 0.14 \\
\hline unclassified_f_Clavicipitaceae & 0.02 & 0.01 & 1.41 & 0.23 \\
\hline Cladophialophora & 1.45 & 0.34 & 0.03 & 0.26 \\
\hline Oidiodendron & 1.04 & 0.67 & 0.02 & 0.31 \\
\hline unclassified_o_Pezizales & 0.04 & 0.02 & 1.51 & 0.15 \\
\hline unclassified_o_Agaricales & 0.02 & 0.01 & 1.32 & 0.08 \\
\hline others & 19.77 & 15.66 & 7.93 & 15.01 \\
\hline
\end{tabular}


Community heatmap analysis on Species level

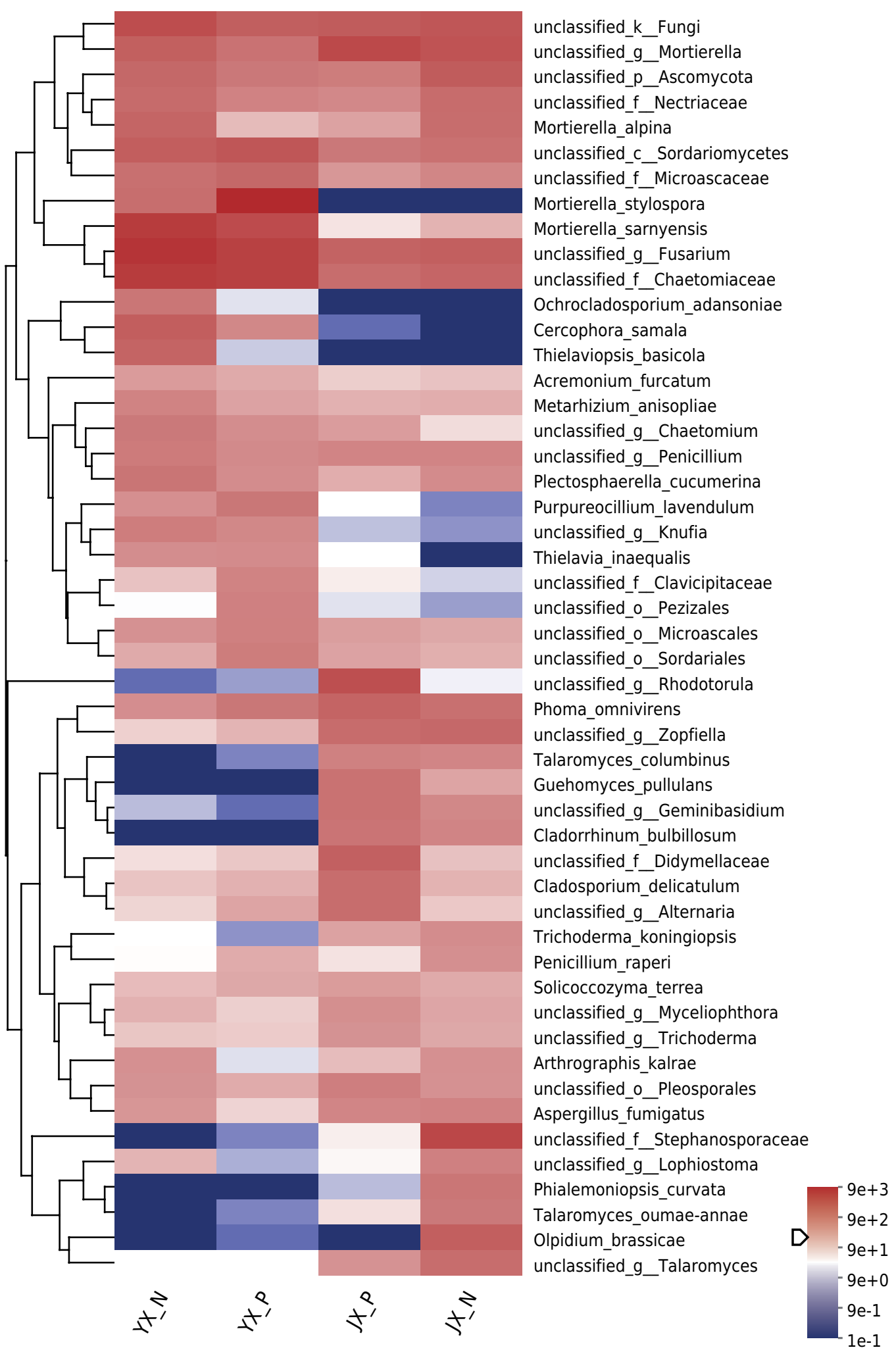

Figure 4. The mcrobial community heatmap on species level in rhizosphere soils at different samples.

In previous studies, the occurrence of RKN disease was closely related to the interaction between soil microbial communities (Echeverrigaray et al., 2010). Studies reported significant differences in the bacterial community richness and diversity between RKN diseased and healthy soil and the presence of different microbial species of the bacteria, but no difference was observed in all aspects of the fungi (Huang et al., 2020). We found 


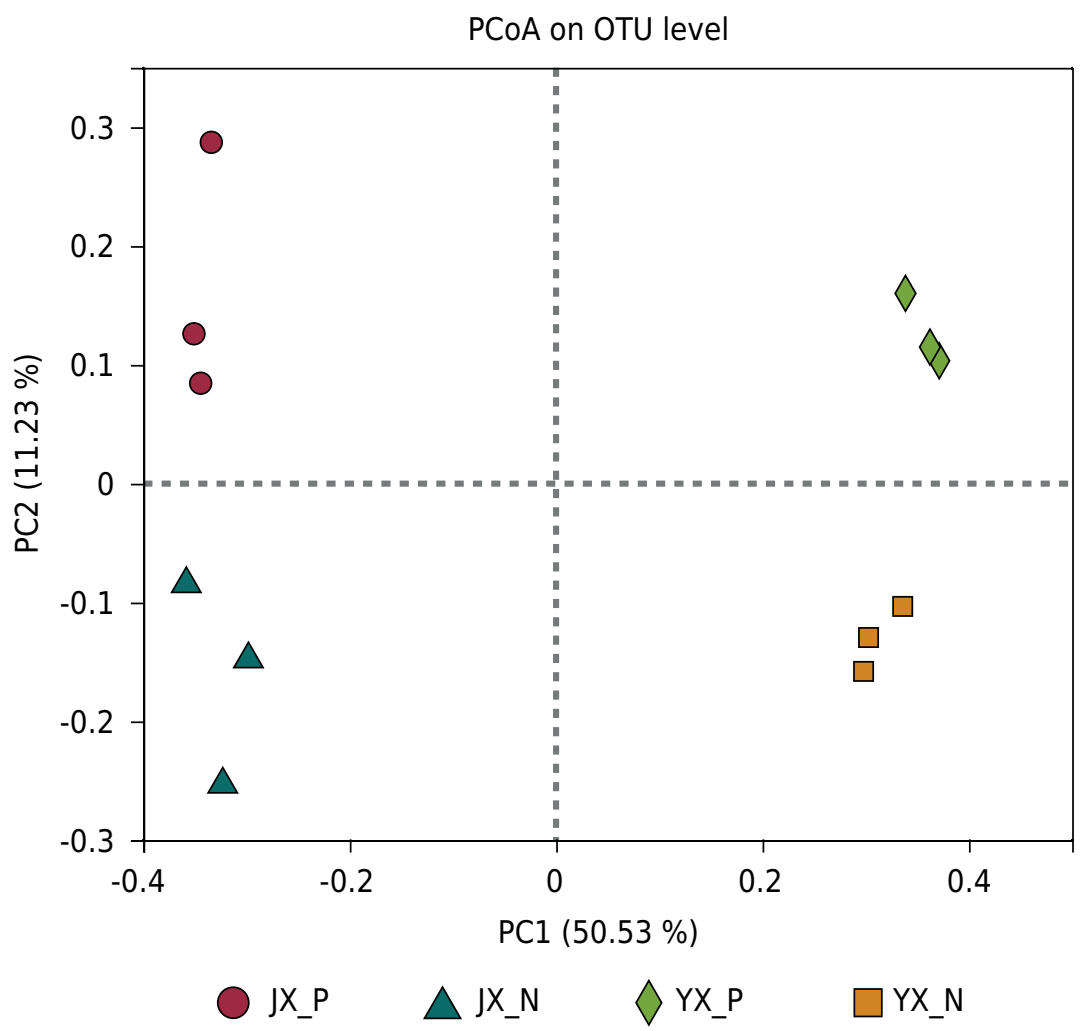

Figure 5. PCoA cluster analysis of fungus community in rhizosphere soils at different samples.

that the soil not infected by Meloidogyne incognita had higher fungal diversity than the infected soil, which indicates that the lower microbial diversity may result in high RKN infection. Microbial diversity is an excellent indicator of soil health. Loss of soil microbial diversity contributes to an increase in soil-borne plant diseases; on the contrary, the high microbial, as well as functional diversity and activity, are involved in plant growth promotion, plant defense, and soil-borne disease suppression (Mendes et al., 2015; Raaijmakers et al., 2016). The scientific community has a great interest in developing strategies that reshape the rhizosphere microbial community to attain stable and more diverse conditions to prevent or mitigate pathogen/pest occurrence (Jaiswal et al., 2017). Thus, knowing what kind of microbes and their role is very important.

We found that in rhizosphere soil samples, the abundances of OTUs in soils from healthy plants were higher than in soils from diseased plants. More beneficial fungi, such as Penicillium and Aspergillus, were found in soil samples of healthy plants, whereas more pathogenic fungi, such as Phoma and Alternaria, were found in soil samples of diseased plants. Some studies have shown that a small class of key groups are present in the rhizosphere microbial community, and they play a leading role in promoting disease occurrence (Berendsen et al., 2012; Lawson et al., 2019). Penicillium participates in the decomposition of organic matter, promotes the cycling of C, N, P and other elements, and degrades a variety of environmentally harmful substances (Luo et al., 2016). Purpureocillium lavendulum is an important nematode biocontrol fungi (Tong et al., 2021). Increases in two kinds of beneficial microorganisms have positive effects on optimizing the rhizosphere fungal community and on improving the micro-ecological environment. Phoma spp. are important plant pathogens that often cause stem rot, branch blight, and leaf and fruit necrosis. Among them, $P$. omnivirens causes phoma disease by infecting tobacco stems and leaves (Jiang et al., 2018). Alternaria is also a common plant pathogen that can infect tobacco, producing brown spots. The relative abundances of these two genera in the rhizosphere soil of diseased tobacco increased, which may be related to the increase in the incidence rates of complex infectious diseases after infection by Meloidogyne spp. (Li et al., 2013). 


\section{CONCLUSIONS}

Occurrence of RKN disease in tobacco was closely related to the fungal community and diversity level in the rhizosphere soil. Soil rhizosphere of healthy tobacco plants showed greater fungal diversity and abundance, and the fungal community was more balanced than that of infected plants. The relative abundance of beneficial fungi, such as Penicillium and Aspergillus in the rhizosphere soil of diseased plants decreased, whereas those of pathogenic fungi, such as Phoma and Alternaria, increased. The imbalance of rhizosphere micro-ecology may further aggravate the disease state of tobacco. Therefore, reshaping stable and diversified rhizosphere microbial communities to enhance the rhizosphere micro-ecological resilience may help to increase tobacco resistance against $M$. incognita, although it is still a challenge to manipulate the soil traits to reach the ideal community structure.

\section{ACKNOWLEDGEMENTS}

This study was supported by the Project of Yunnan Branch Company of China Tobacco Corporation (No. 2018530000241016, 2019530000241011, 2018530000241020, and 2020530000242010).

\section{AUTHOR CONTRIBUTIONS}

Conceptualization: (D) Huang Feiyan (lead).

Data curation: (D) Gao Lingling (equal) and (D) Yang Min (equal).

Investigation: (D) Wang Jianwel (lead).

Methodology: (D) Deng Xiaopeng (equal) and (D) Zhang Zhan (equal).

Project administration: (D) Yu Lei (lead).

Resources: (D) Yang Yongfeng (lead).

Software: (D) Cai Yongzhan (lead).

Writing - original draft: (D) Chen Xiaolong (lead).

Writing - review \& editing: (D) Tong Wenjie (lead).

\section{REFERENCES}

Altschul SF, Gish W, Miller W, Myers EW, Lipman DJ. Basic local alignment search tool. J Mol Biol. 1990;215:403-10. https://doi.org/10.1016/S0022-2836(05)80360-2

Back MA, Haydock PPJ, Jenkinson P. Disease complexes involving plant parasitic nematodes and soilborne pathogens. Plant Pathol. 2002;1:683-67. https://doi.org/10.1046/j.1365-3059.2002.00785.x

Berendsen RL, Pieterse CM, Bakker PA. The rhizosphere microbiome and plant health. Trends Plant Sci. 2012;7:478-46. https://doi.org/10.1016/j.tplants.2012.04.001

Bowen JL, Kearns PJ, Byrnes J, Wigginton S, Allen WJ, Greenwood M, Tran K, Yu J, Cronin JT, Meyerson LA. Lineage overwhelms environmental conditions in determining rhizosphere bacterial community structure in a cosmopolitan invasive plant. Nat Commun. 2017;8:433. https://doi.org/10.1038/s41467-017-00626-0

Cao Y, Tian B, Ji X, Shang S, Lu C, Zhang K. Associated bacteria of different life stages of Meloidogyne incognita using pyrosequencing-based analysis. J Basic Microbiol. 2015;55:950-60. https://doi.org/10.1002/jobm.201400816 
Caporaso JG, Lauber CL, Walters WA, Berg-lyons D, Lozupone CA, Turnbaugh PJ, Fierer N, Knight R. Global patterns of 16S rRNA diversity at a depth of millions of sequences per sample. Proc Natl Acad Sci. 2011;108:4516-22. https://doi.org/10.1073/pnas.1000080107

Chen ZB, Xia ZY, Xu SG, Wang HY, Zhao XN, Li JW. Screening of tobacco endophytic bacteria resistant to Meloidogyne spp and its control effect. Acta Tab Sin. 2015;21:71-5. https://doi.org/10.16472/j.chinatobacco.2014.095

Ciancio A, Colagiero M, Pentimone I, Rosso L. Soil microbial communities and their potential for root-knot nematodes management: a review. Environ Eng Manage J. 2016;15:1833-9. https://doi.org/10.30638/eemj.2016.195

Cui JK, Ren HH, Meng HG, Chang D, Jiang SJ. Research progress on the occurrence and control of tobacco root knot nematode in China. Acta Phytopathol Sin. 2021;51:663-82. https://doi.org/10.13926/j.cnki.apps.000487

Echeverrigaray S, Zacaria J, Beltrão R. Nematicidal activity of monoterpenoids against the root-knot nematode Meloidogyne incognita. Phytopathology. 2010;100:199-203. https://doi.org/10.1094/PHYTO-100-2-0199

Edgar RC. UPARSE: Highly accurate OTU sequences from microbial amplicon reads. Nat Methods. 2013;10:996-8. https://doi.org/10.1038/nmeth.2604

Haegeman A, Vanholme B, Jacob J, Vandekerckhove TT, Claeys M, Borgonie G, Gheysen G. An endosymbiotic bacterium in a plant-parasitic nematode: member of a new wolbachia supergroup. Int J Parasitol. 2009;39:1045-54. https://doi.org/10.1016/j.ijpara.2009.01.006

Huang K, Jiang Q, Liu L, Zhang S, Zhang Y. Exploring the key microbial changes in the rhizosphere that affect the occurrence of tobacco root-knot nematodes. AMB Express. 2020;10:72. https://doi.org/10.1186/s13568-020-01006-6

Jaiswal AK, Elad Y, Paudel I, Graber ER, Cytryn E, Frenkel O. Linking the belowground microbial composition, diversity and activity to soilborne disease suppression and growth promotion of tomato amended with biochar. Sci Rep. 2017;7:44382. https://doi.org/10.1038/srep44382

Jeger M, Bragard C, Cafer D, Candresse T, Chatzivassiliou E, Dehne-Schmutz K, Gilioli G, Grégoire JC, Miret JAJ, MacLeod A, Navarro MN, Parnell S, Potting R, Rafoss T, Ross V, Urek G, Van Bruggen A, Werf WV, West J, Winter S, Kaluski T, Niere B. Pest categorisation of Nacobbus aberrans. EFSA J. 2018;16:5249. https://doi.org/10.2903/j.efsa.2018.5249

Jiang Y, San WJ, Zeng EL, Wang Y, Wang DF, Qin K. Occurrence and pathogen identification of tobacco stem spot disease in Guizhou Province. Jiangsu Agric Sci. 2018;46:92-5. https://doi.org/10.15889/j.issn.1002-1302.2018.10.024

Kim YC, Anderson AJ. Rhizosphere pseudomonads as probiotics improving plant health. Mol Plant Pathol. 2018;10:231-43. https://doi.org/10.1111/mpp.12693

Lawson CE, Harcombe WR, Hatzenpichler R, Lindemann SR, Löfer FE, O’Malley MA, Martín HG, Pfeger BF, Raskin L, Venturelli OS, Weissbrodt DG, Noguera DR, McMahon KD. Common principles and best practices for engineering microbiomes. Nat Rev Microbiol. 2019;17:725-41. https://doi.org/10.1038/s41579-019-0255-9

Li F, Zhu X, Qiao F, Chen XF, Li H, Hu JF. psoRPM1 gene from Prunus sogdiana indicated resistance to root-knot nematode in tobacco. Acta Hortic Sin. 2013;40:2497-504. https://doi.org/10.16420/j.issn.0513-353x.2013.12.019

Li HL, Wang SZ. Relationship between rhizosphere microbe and plant disease. Acta Agric Univ Henanensis. 1989;4:401-8. https://doi.org/10.16445/j.cnki.1000-2340.1989.04.011

Liu X, Zhang S, Jiang Q, Bai Y, Shen G, Li S, Ding W. Using community analysis to explore bacterial indicators for disease suppression of tobacco bacterial wilt. Sci Rep. 2016;6:36773. https://doi.org/10.1038/srep36773

Liu XL. Infection mechanism of Paecilomyces lilacinus strains on Meloidogyne incognita. Anhui Agric Univ. 2011.

Luo Q, Peng C, Ye BP. New advances in research of the genus Penicillium. Pharm Biotechnol. 2016;23:452-6. https://doi.org/10.19526/j.cnki.1005-8915.2016.05.017 
Mendes LW, Tsai SM, Navarrete AA, Hollander MD, Veen JAV, Kuramae EE. Soil-borne microbiome: Linking diversity to function. Microb Ecol. 2015;70:255-65. https://doi.org/10.1007/s00248-014-0559-2

Norabadi MT, Sahebani N, Etebarian HR. Biological control of root-knot nematode (Meloidogyne javanica) disease by Pseudomonas fluorescens (Chao). Arch Phytopathol Plant Prot. 2014;47:615-21. https://doi.org/10.1080/03235408.2013.816102

Raaijmakers JM, Mazzola M. Soil immune responses soil microbiomes may be harnessed for plant health. Science. 2016;352:1392-3. https://doi.org/10.1126/science.aaf3252

Ruby EG. Symbiotic conversations are revealed under genetic interrogation. Nat Rev Microbiol. 2008;6:752-62. https://doi.org/10.1038/nrmicro1958

Siddiqui IA, Shaukat SS, Hamid M. Role of zinc in rhizobacteria-mediated suppression of root-infecting fungi and root-knot nematode. J Phytopathol. 2002;150:569-75. https://doi.org/10.1046/j.1439-0434.2002.00805.x

Stirling GR, Rames E, Stirling AM, Hamill S. Factors associated with the suppressiveness of sugarcane soils to plant-parasitic nematodes. J Nematol. 2011;43:135-48. https://doi.org/10.1002/jez.b.21423

Tian BY, Cao Y, Zhang KQ. Metagenomic insights into communities, functions of endophytes, and their associates with infection by root-knot nematode, Meloidogyne incognita, in tomato roots. Sci Rep. 2015;5:17087. https://doi.org/10.1038/srep17087

Tong WJ, Deng XP, Xu ZL, Ma ED, Jin Y, Li JY. Effect of plowing depth on soil physical characteristics and spatial distribution of root system of flue-cured tobacco. Chin J Eco-Agric. 2016;24:1464-72. https://doi.org/10.13930/j.cnki.cjea.160555

Tong WJ, Yang M, Wang H, Feng XB, Zhang LC, Zhou B, Chen F, Huang FY, Chen XL, Cai YZ, Yu $L$, Deng XP. Effects of different tillage methods on bacterial community and enzyme activity of rhizosphere of flue-cured tobacco in Yunnan mountains. Int J Agric Biol. 2021;25:345-53. https://doi.org/10.17957/IJAB/15.1674

Wang Q, Garrity GM, Tiedje JM, Cole JR. Naive bayesian classifier for rapid assignment of rRNA sequences into the new bacterial taxonomy. Appl Environ MicroBiol. 2007;73:5261-7. https://doi.org/10.1128/AEM.00062-07

Yang SJ, Lei LP, Zhu ML, Xia ZY, Li YH. Screening of the parasitical fungi of root knot nematode in tobacco. Southwest Chin J Agric Sci. 2004;17:151-4. https://doi.org/10.16213/j.cnki.scjas.2004.s1.036

Yang Z, Dai CC, Wang XX, Li XG. Advance in research on rhizosphere microbial mechanisms of crop soil-borne fungal diseases. Acta Pedol Sin. 2019;56:12-22. https://doi.org/10.11766/trxb201803260058

Zhang T, Wang NF, Liu HY, Zhang YQ, Yu LY. Soil pH is a key determinant of soil fungal community composition in the Ny-Ålesund region, Svalbard (high arctic). Front Microbiol. 2016; 7:227. https://doi.org/10.3389/fmicb.2016.00227 